\title{
sciendo
}

\section{Effects of an Exogenous Ketone Supplement on Five-Kilometer Running Performance}

\author{
by \\ Philip J. Prins ${ }^{1}$, Andrew P. Koutnik ${ }^{2}$, Dominic P. D'Agostino², \\ Christopher Q. Rogers' ${ }^{2}$ Jacob F. Seibert ${ }^{1}$, Jillian A. Breckenridge, \\ Daniel S. Jackson ${ }^{1}$, Edward J. Ryan ${ }^{3}$, Jeffrey D. Buxton ${ }^{1}$, Dana L. Ault ${ }^{1}$
}

Numerous oral ketone supplements are marketed with the claim that they will rapidly induce ketosis and improve exercise performance. The purpose of this study was to assess exercise performance time and related physiological, metabolic and perceptual responses of recreational endurance runners after ingestion of a commercially available oral ketone supplement. Recreational endurance runners ( $n=10$; age: $20.8 \pm 1.0$ years; body mass: $68.9 \pm 5.6$ $\mathrm{kg}$; height: $175.6 \pm 4.9 \mathrm{~cm})$ participated in a double-blind, crossover, repeated-measures study where they were randomized to $300 \mathrm{mg} . \mathrm{kg}-1$ body weight of an oral $\beta$-hydroxybutyrate-salt + Medium Chain Triglyceride ( $\beta H B-$ salt $+M C T$ ) ketone supplement or a flavor matched placebo (PLA) 60 min prior to performing a 5-km running time trial (5KTT) on a treadmill. Time, HR, RPE, affect, RER, VO,$V C O_{2}$, and VE were measured during the 5-km run. The Session RPE and affect (Feeling Scale) were obtained post-5KTT. Plasma glucose, lactate and ketones were measured at baseline, 60-min post-supplement, and immediately post-5KTT. Plasma R- $\beta H B$ (endogenous isomer) was elevated from baseline and throughout the entire protocol under the $\beta H B-$ salt $+M C T$ condition $(p<0.05)$. No significant difference ($58.3 \pm 100.40$ s; $95 \%$ CI: $-130.12-13.52 ; p=0.100)$ was observed between the $\beta H B$-salt $+M C T$ supplement $(1430.0 \pm$ $187.7 s)$ and the PLA $(1488.3 \pm 243.8 s)$ in time to complete the 5KTT. No other differences $(p>0.05)$ were noted in any of the other physiological, metabolic or perceptual measures.

Key words: ketosis, beta-hydroxybutyrate, dietary supplements, ergogenic aid.

\section{Introduction}

Ketosis is defined as elevated circulating levels of ketone bodies (beta-hydroxybutyrate; $\beta \mathrm{HB}$, acetoacetate, acetone)]. Traditionally, ketosis was achieved through fasting (Cahill, 2006), a very low-carbohydrate ketogenic diet (VLCKD; Phinney et al., 1980, 1983a, 1983b), or under pathological conditions where insulin levels were insufficient to prevent rapid ketogenesis (i.e. mismanaged Type-1 Diabetes). The VLCKD usually does not require caloric restriction and was originally used as an effective dietary replacement to fasting due to its overlapping mechanisms (Newman and Verdin, 2017) for epileptic seizures (Paoli et al., 2013). Currently, the VLCKD is being explored as a metabolic tool for other neurological disorders, obesity, inflammatory disorders, Type- 1 and -2 diabetes mellitus, polycystic ovarian syndrome, and cancer, amongst others (Koutnik et al., 2019; Paoli et al., 2013; Poff et al., 2017; Stafstrom and Rho, 2012). The VLCKD has also been investigated for longevity (Newman et al., 2017; Roberts et al., 2017), along with cognitive and physical performance (Newman et al., 2017; Volek et al., 2016; Wilson et al., 2017).

1 - Department of Exercise Science, Grove City College, Grove City, PA, USA.

2 - Department of Molecular Pharmacology \& Physiology, University of South Florida, USA. 
Emerging interest into the metabolic influence of the VLCKD on athletic performance began in the early 1980s where untrained and trained athletes were able to maintain or improve performance even in the absence of sufficient dietary carbohydrates (Phinney et al., 1980, 1983a). Since that time, numerous studies have explored the metabolic influence of diet on aerobic (Zajac et al., 2014) and anaerobic (Michalczyk et al., 2019; Paoli et al., 2012; Wilson et al., 2017) performance to determine if major shifts in substrate metabolism translate to altered performance outcomes. However, lifestyle modifications can be difficult for some to maintain (Dansinger et al., 2005). Simultaneously, interest has arisen to develop forms of ketone bodies that can be exogenously administered. Several exogenous ketone formulations have been developed including R- $\beta \mathrm{HB}+\mathrm{S}-\beta \mathrm{HB}$-salt $(\beta \mathrm{HB}-$ salt), $\beta$ HB-salt + Medium Chain Triglycerides $(\beta \mathrm{HB}$-salt+MCT), and ketone esters, each of which elevated circulating levels of ketone bodies when orally administered (Kesl et al., 2016). Comparative analysis in human subjects also demonstrated the ability of exogenous ketones to increase circulating ketone levels (Stubbs et al., 2017). With the emergence of commercially available exogenous ketone formulations, research has focused on exploring their use in athletic performance (Cox et al., 2016; Evans and Egan, 2018; Evans et al., 2018).

Exogenous ketone supplements that have been used to manipulate metabolism in studies investigating athletic performance can be divided into two categories: Ketone Esters (Cox et al., 2016; Evans and Egan, 2018; Leckey et al., 2017) and $\beta$ HB-salts (Evans et al., 2018; O'Malley et al., 2017; Rodger et al., 2017; Waldman et al., 2018), with the former inducing greater elevations in $\beta \mathrm{HB}$ with correlated changes in systemic metabolism (Stubbs et al., 2017). To date, one trial showed exercise improvements (Cox et al., 2016), three trials showed neutral effects (Evans and Egan, 2018; Rodger et al., 2017; Waldman et al., 2018), and two trials showed negative effects using exogenous ketone supplementation (Leckey et al., 2017; O'Malley et al., 2017). The likely explanation for divergent findings is the heterogeneity of exercise duration, task, and intensity, along with divergent ketone formulations and dose. Additionally, most trials involve cycling protocols (Cox et al., 2016; Leckey et al., 2017; O'Malley et al., 2017; Waldman et al., 2018; Rodger et al., 2017). Thus, further research into the ergogenic or ergolytic potential of various forms of ketone supplementation in other performance contexts is warranted.

Interestingly, all $\beta \mathrm{HB}$-salt trials done thus far have utilized varying dosages (140-536 $\mathrm{mg} / \mathrm{kg}$ ), but resulted in similar elevations in R$\beta \mathrm{HB}$ (Evans et al., 2018; O'Malley et al., 2017; Rodger et al., 2017; Waldman et al., 2018), indicating that gut absorption may be a limiting factor in rapidly raising $\mathrm{R}-\beta \mathrm{HB}$ with $\beta \mathrm{HB}$-salts. However, Kesl et al. (2016) found that a $\beta \mathrm{HB}$ salt+MCT combination prolonged $\mathrm{R}-\beta \mathrm{HB}$ elevation in rats, and this was observed to be a superior formula. Additionally, it has been previously reported that MCTs are rapidly metabolized to R- $\beta$ HB (Bach et al., 1996; Seaton et al., 1986) and produce a more robust thermogenic effect than other dietary fat sources (Mascioli et al., 1991; Seaton et al., 1986). However, the $\beta$ HBsalt+MCT combination has never been explored for exercise performance and ketone supplementation has not been applied to a single intermediate distance running bout. Consequently, we sought to explore the ability of a commercially available exogenous ketone mixture of $\beta \mathrm{HB}$-salt+MCT to impact physiologic, metabolic, perceptional, and performance responses to intermediate distance running performance in a randomized, cross-over design in recreational, college-age endurance athletes. We hypothesized that this $\beta \mathrm{HB}$-salt+MCT drink would alter blood metabolites and metabolism, but neither perception nor athletic performance, in line with previous work exploring $\beta \mathrm{HB}$-salt alone (Evans et al., 2018; O'Malley et al., 2017; Rodger et al., 2017; Stubbs et al., 2017; Waldman et al., 2018).

\section{Methods}

\section{Experimental Approach to the Problem}

A double-blind placebo-controlled crossover design was employed consisting of one familiarization trial and two experimental trials. During the familiarization session, participants were informed of all procedures and familiarized with all performance measures to reduce the possibility of a learning effect. The familiarization trial was identical to experimental trials except 
that participants consumed no supplement prior to exercise. After familiarization, participants completed two experimental conditions (ketone or placebo), the order of which was completed in a randomized and counterbalanced fashion separated by 7 days. On experimental days, participants consumed either $300 \mathrm{mg} \cdot \mathrm{kg}^{-1}$ body weight of the ketone supplement ( $\beta \mathrm{HB}$-salt+MCT) or a flavor matched placebo (PLA) $60 \mathrm{~min}$ prior to performing a $5-\mathrm{km}$ running time trial on a treadmill. Plasma glucose and ketones were measured at baseline, $60 \mathrm{~min}$ post supplement ingestion, and immediately following the time trial. Plasma blood lactate concentration was measured before and after the time trial. Other variables evaluated were (a) $5-\mathrm{km}$ running time, (b) RPE (RPE-Overall; RPE-Chest; RPE-Legs), (c) heart rate, (d) affect, (e) session RPE, (f) session affect, and (g) 500-m split times during the 5-km time trial. The RPE, heart rate, and affect were taken every $500-\mathrm{m}$ during the $5-\mathrm{km}$ time trial. In addition, during the time trial oxygen uptake $\left(\mathrm{VO}_{2}\right)$, carbon dioxide production $\left(\mathrm{VCO}_{2}\right)$, minute ventilation $\left(\mathrm{V}_{\mathrm{E}}\right)$ and respiratory exchange ratio (RER), were assessed and derived from indirect calorimetry. Testing sessions were conducted at the same time each day at a room temperature between $19-21^{\circ} \mathrm{C}$ and a relative humidity of 35$40 \%$. All testing took place at the Exercise Science Laboratory of Grove City College.

\section{Participants}

Ten male, recreational runners (age: $20.8 \pm$ 1.03 years; body height: $175.6 \pm 4.9 \mathrm{~cm}$; body mass: $68.9 \pm 5.6 \mathrm{~kg}$; body fat: $13.4 \pm 3.6 \%$; lean body mass: $59.6 \pm 3.1 \mathrm{~kg}$; body mass index: $22.4 \pm 1.9$ $\mathrm{kg} / \mathrm{m}^{2}$; running distance per week: $34.6 \pm 5.5 \mathrm{~km}$ ) volunteered to participate in this study. Participants were recruited directly from local running clubs, and by advertising within the local community. Included men: (a) currently run an average of $32 \mathrm{~km}$ per week; (b) were between the ages of 18-35; and (c) had completed a $5-\mathrm{km}$ distance run under $30 \mathrm{~min}$ within the last 3 months. Participants were not currently taking medications or following a low-carbohydrate or ketogenic diet, or currently consuming nutritional ketone supplements. Participants were prohibited from using any ergogenic aids for one month preceding the study and were asked to refrain from taking any performance enhancing supplement(s) other than the experimental beverage during the study. Runners were instructed not to change their regular exercise training and dietary habits for the duration of the study, and they were told not to exercise prior to testing. Participants were instructed to refrain from caffeine, and alcohol consumption for 48 hours, physical activity for 24 hours, and food and drink for 3 hours before each exercise test (Astorino et al., 2012; Forbes et al., 2007). Participants were instructed to maintain a training and dietary $\log$ for 3 days before the first experimental trial. They were provided with a copy of their pre-trial log, and were instructed to have the same dietary intake and physical activity during the 48 hours before the other trials. Dietary logs were checked to ensure compliance and we were satisfied that all of the participants adhered to the instructions given and that the food and drinks consumed before each trial were the same. Before enrolling in the investigation, participants were fully informed of any risks and discomforts associated with the experiments prior to giving their written informed consent to participate. The experimental protocol was approved by the Institutional Review Board of Grove City College prior to implementation.

\section{Procedures}

Familiarization Testing. At the first laboratory visit, all the experimental procedures were explained to the participants. Participants underwent an orientation involving practice of treadmill running and familiarization of the various experimental instruments, equipment, affect and perceived exertion. Affect was measured using a validated 11-point Feeling Scale (Hardy, 1989) with participants informed that their responses should reflect the affective or emotional components of the exercise and not the physical sensation of effort or strain. The OMNI Walk/Run Perceived Exertion Scale (Robertson et al., 2004) was used to measure the physical perceptions of exertion for overall body (RPE-O), legs (RPE-L) and chest (RPE-C). Following the orientation session, anthropometric measures were obtained including body height $(\mathrm{cm})$, body mass $(\mathrm{kg})$, fat free mass $(\mathrm{kg})$ and fat mass $(\%$ and $\mathrm{kg})$. Body height $(\mathrm{cm})$ was measured using a physician's scale (Detecto, Webb City, MO). Body mass $(\mathrm{kg})$ and body composition (fat and lean mass) were estimated using a Tanita bioelectrical impedance analyzer (BIA) (TBF-310GS Tanita 
Corporation of America, Arlington Heights, Illinois).

Experimental Trials. On experimental testing days, participants were given either a commercially available $\beta \mathrm{HB}$-salt+MCT or a flavormatched PLA. The beverages were served cold ( 6 ${ }^{\circ} \mathrm{C}$ ) and were provided to participants $60 \mathrm{~min}$ before the test began. Participants were instructed to drink the beverage within $2 \mathrm{~min}$ of receiving it. To ensure a double-blinded design, each drink was presented to participants in an opaque sports bottle. After consumption of the beverage, participants were instructed to sit quietly for 1 hour.

Before the start of the time trial, participants completed a 5-min warm-up run on the treadmill (TMX425C treadmill; Trackmaster, Newton, KS, USA). Immediately thereafter, the treadmill was brought to a standstill $\left(0 \mathrm{~km} \cdot \mathrm{hr}^{-1}\right)$. At this point all timing devices were reset, the distance covered on the treadmill monitor was reset, and a 5-s count down was given to signal that the time trial has begun (Prins et al., 2016). Participants were instructed to finish the $5-\mathrm{km}$ run as fast as possible on a motorized treadmill at $0.0 \%$ grade. Participants were provided with feedback on the distance (at regular 500-m intervals) covered during each $5-\mathrm{km}$ time trial and were not informed of the overall performance time until completion of the final testing session. The heart rate (Polar Electro, Kempele, Finland), RPE (RPE-Overall; RPE-Chest; RPE-Legs) and affect (Feeling Scale) were recorded at 500-m intervals during the time trial. During the time trial, participants were allowed to adjust their pace via control buttons located on the treadmill. The speed indicator was concealed from the participant's view throughout the time trial. Therefore, participants regulated their treadmill pace according to their perceived exertion associated with the intensity of the exercise and their subjective feelings of their running capabilities. During each treadmill run all timing devices were removed from the participant's sight. Immediately upon cessation of treadmill exercise, participants estimated their perceived exertion and affect, and a final heart rate measurement was taken. Lastly, ratings of perceived exertion and affect for the entire exercise session (session RPE and session affect) were obtained $5 \mathrm{~min}$ following the time trial. Gas samples were collected throughout the time trial using a metabolic cart (TrueOne $2400 \AA$ Metabolic Measurement System, Parvo Medics, Inc., Sandy, UT). To establish any learning effect after completion of the two experimental trials, $5-\mathrm{km}$ time to completion was compared between the supplement and the placebo trial using a paired samples t-test. No significant difference between trials was observed $(p=0.577)$. In addition, intraclass correlation coefficients were computed for all conditions of all participants of the 5KTT and was reported to be ICC $r=0.870$ with a $95 \%$ confidence interval of 0.584-0.965.

Metabolic Gases. Prior to each 5KTT, opencircuit spirometry was calibrated with room air and gases of known concentrations, which was used to estimate $\mathrm{VO}_{2}\left(\mathrm{ml}^{\mathrm{kg}}{ }^{-1} \cdot \mathrm{min}^{-1}\right)$ by sampling and analyzing breath-by-breath expired gases. Expired gases $-\mathrm{VO}_{2}, \mathrm{VCO}_{2}, \mathrm{VE}$, and $\mathrm{RER}$, were monitored continuously during exercise and expressed as 30-s averages.

Blood Sampling. Finger-tip blood samples were collected and immediately processed for plasma blood lactate (Lactate Plus, Nova Biomedical), ketones $(R-\beta \mathrm{HB}$; Precision Xtra, Abbott Diabetes Care Inc., Almeda, CA) and glucose (Precision Xtra, Abbott Diabetes Care Inc., Almeda, CA) concentrations. Fingertip capillary samples were collected using a lancet following cleaning with alcohol swab and dried. The first droplet was wiped away with a cotton swab to remove any alcohol and the subsequent droplets were used for analysis. Blood samples were measured at baseline, $60 \mathrm{~min}$ post-supplement ingestion, and at the end of the time trial.

Supplementation. The supplement used in this study consisted of $\beta \mathrm{HB}$-salt+Medium Chain Triglyceride (KETO//OS 2.1 Orange Dream, Pruvit, Melissa, TX, USA). Participants consumed $300 \mathrm{mg} \cdot \mathrm{kg}^{-1}$ of body mass of the ketone supplement powder mixed with approximately $237 \mathrm{ml}$ of cold $\left(\sim{ }^{\circ} \mathrm{C}\right)$ water prior to ingestion. The supplement contained a calculated 9.0 $\beta \mathrm{HB}$ racemic salt $(50 \% R-\beta \mathrm{HB}$ and $50 \% \quad S-\beta \mathrm{HB})$ and reported $7 \mathrm{~g}$ MCT. A complete list of the ingredients and the relative dose of each ingredient is provided in Table 1. Volume for the drinks was measured with a graduated cylinder and the dry ingredients were measured to the nearest $0.001 \mathrm{~g}$ on a calibrated balance scale (Denver Instrument, Bohemia, NY). When 
receiving the PLA, participants consumed an equal amount of water with $\mathrm{MiO}$ Orange Tangerine Liquid Enhancer ( $0 \mathrm{mg}$ caffeine, $0 \mathrm{kcal}$; Kraft Foods; MiO, Northfield, IL, USA). The ketone supplement, and PLA drink were similar in volume, texture, and appearance. The taste of the drinks was slightly different, and there remains the possibility that participants were able to identify the drinks. To avoid the placebo effect in the experimental trials, we did not inform participants about the names of the drinks and we presented both drinks as having similar ergogenic properties.

\section{Statistical Analysis}

Statistical analyses were performed using SPSS version 24.0 (SPSS Inc., Chicago, IL). Statistical significance was set a priori at $p<0.05$. Descriptive statistics were calculated for all variables. Data were tested for normality using the Shapiro-Wilk test. Performance data $(5-\mathrm{km}$ running time), mean exercise heart rate, RER, $\mathrm{VO}_{2}, \mathrm{VCO}_{2}, \mathrm{VE}_{\mathrm{E}}$, affect, RPE-Chest, RPE-Legs, RPEOverall, session RPE and session affect were analyzed using a paired-samples $t$-Test. A $2 \times 10$ (condition $\mathrm{x}$ distance during the $5-\mathrm{km}$ time trial) repeated measures analysis of variance was conducted to assess the effect of time, treatment, and interaction between time and treatment, on heart rate, affect, RPE-Chest, RPE-Legs, RPEOverall, and time covered at each 500-m interval during the $5-\mathrm{km}$ time trial. A 2 (condition, supplement vs placebo) × 3 (rest, 60 min post ingestion, and at end of exercise) repeated measures analysis of variance was conducted to assess the effect of time, treatment, and interaction between time and treatment, on plasma glucose and ketones. A $2 \times 2$ (before and after exercise) repeated measures analysis of variance was conducted to assess the effect of time, treatment, and interaction between time and treatment, on plasma lactate. The assumption of sphericity was confirmed using Mauchly's test. GreenhouseGeisser epsilon corrections were used when the sphericity assumption was violated. Effect sizes (Cohen's d) were calculated and interpreted as: small effect $>2$; medium effect $>0.5$; large effect $>$ 0.8 .

\section{Results}

\section{Time Trial Performance}

No significant difference $(p=0.100)$ was observed between the $\beta \mathrm{HB}$-salt+MCT supplement $(1430.0 \pm 187.7 \mathrm{~s})$ and the PLA $(1488.3 \pm 243.8 \mathrm{~s})$ in time to complete the 5KTT (Figure 1; Table 2). However, in comparison to the mean time for the PLA trial, $\beta \mathrm{HB}$-salt+MCT supplement ingestion resulted in an average non-significant decrease in time to completion of $58.3 \mathrm{~s}(4.06 \%$; $95 \%$ CI: 130.12 - 13.52; ES = 0.27). The individual range in reduced time to completion across participants was 43 - 214 s. Eight participants $(80 \%)$ ran faster after consuming the $\beta \mathrm{HB}$-salt+MCT supplement, while two participants $(20 \%)$ ran faster after consuming the PLA.

Table 1

Ingredients contained in 1 dose of the ketone supplement in absolute serving size

\begin{tabular}{lc}
\hline Ingredient & Amount per serving \\
\hline Total Calories & $110 \mathrm{Kcal}$ \\
$\beta$-hydroxybutyrate $(\beta \mathrm{HB})$ & $9.0 \mathrm{~g}^{*}$ \\
Medium-Chain Triglycerides (MCT) & $7 \mathrm{~g}$ \\
Sodium & $1.4 \mathrm{~g}$ \\
Potassium & $47 \mathrm{mg}$ \\
Total Carbohydrates & $3 \mathrm{~g}$ \\
& $2 \mathrm{~g}$ \\
Sugars & $1 \mathrm{~g}$ \\
Protein & \\
\hline ata are reported values based on product labeling. Note. ${ }^{*}=$ estimated grams of $\beta$ - \\
ybutyrate ( $\beta$ HB) from 1$)$ calculation of the molecular equivalence of the mineral load \\
HB) and 2) calculation of the difference between total product weight and non- $\beta$ HB \\
\\
ingredient weights.
\end{tabular}


Table 2

Physiological, metabolic and perceptual data for 5- $\mathrm{km}$ time-trial performance between the supplement and the placebo $(N=10)$

\begin{tabular}{|c|c|c|c|c|}
\hline Variable & $\begin{array}{c}\text { Ketone } \\
\text { Supplement }\end{array}$ & Placebo & $p$-value & Effect Size \\
\hline TT performance (s) & $1430.0 \pm 187.7$ & $1488.3 \pm 243.8$ & 0.100 & 0.27 \\
\hline $\mathrm{HR}\left(\mathrm{b} \cdot \mathrm{min}^{-1}\right)$ & $173.1 \pm 15.7$ & $178.8 \pm 7.9$ & 0.322 & 0.46 \\
\hline RPE- O & $5.5 \pm 1.3$ & $5.3 \pm 1.4$ & 0.593 & 0.15 \\
\hline RPE- C & $5.0 \pm 1.4$ & $5.0 \pm 1.3$ & 1.000 & 0.00 \\
\hline RPE- L & $5.3 \pm 1.5$ & $5.0 \pm 1.6$ & 0.434 & 0.19 \\
\hline Affect & $2.3 \pm 1.8$ & $1.8 \pm 1.8$ & 0.143 & 0.28 \\
\hline $\mathrm{VO}_{2}\left(\mathrm{~L} \cdot \mathrm{min}^{-1}\right)$ & $3.0 \pm 0.5$ & $3.0 \pm 0.5$ & 0.665 & 0.08 \\
\hline $\mathrm{VO}_{2}\left(\mathrm{ml} \cdot \mathrm{kg} \cdot \mathrm{min}^{-1}\right)$ & $44.2 \pm 5.6$ & $43.8 \pm 5.9$ & 0.695 & 0.07 \\
\hline $\mathrm{VCO}_{2}\left(\mathrm{~L} \cdot \mathrm{min}^{-1}\right)$ & $3.0 \pm 0.6$ & $2.9 \pm 0.6$ & 0.521 & 0.12 \\
\hline $\mathrm{VE}_{\mathrm{E}}\left(\mathrm{L} \cdot \mathrm{min}^{-1}\right)$ & $70.9 \pm 14.8$ & $69.4 \pm 15.0$ & 0.607 & 0.10 \\
\hline RER & $0.99 \pm 0.04$ & $0.98 \pm 0.04$ & 0.655 & 0.24 \\
\hline Session RPE & $6.2 \pm 1.3$ & $7.3 \pm 1.6$ & 0.093 & 0.76 \\
\hline Session Affect & $2.6 \pm 1.6$ & $2.0 \pm 2.3$ & 0.456 & 0.3 \\
\hline
\end{tabular}

Data are Mean $\pm S D$. Note. $T T=$ time trial; $H R=$ heart rate; $R P E-O=R P E$ for overall body; $R P E-C=R P E$ for chest; $R P E-L=R P E$ for legs; $R P E=$ rating of perceived exertion (OMNI rating of exertion); $R E R=$ Respiratory exchange ratio. Effect sizes, $<0.2=$ trivial; 0.2 to $0.49=$ small;

0.5 to $0.79=$ moderate; $>0.8=$ large

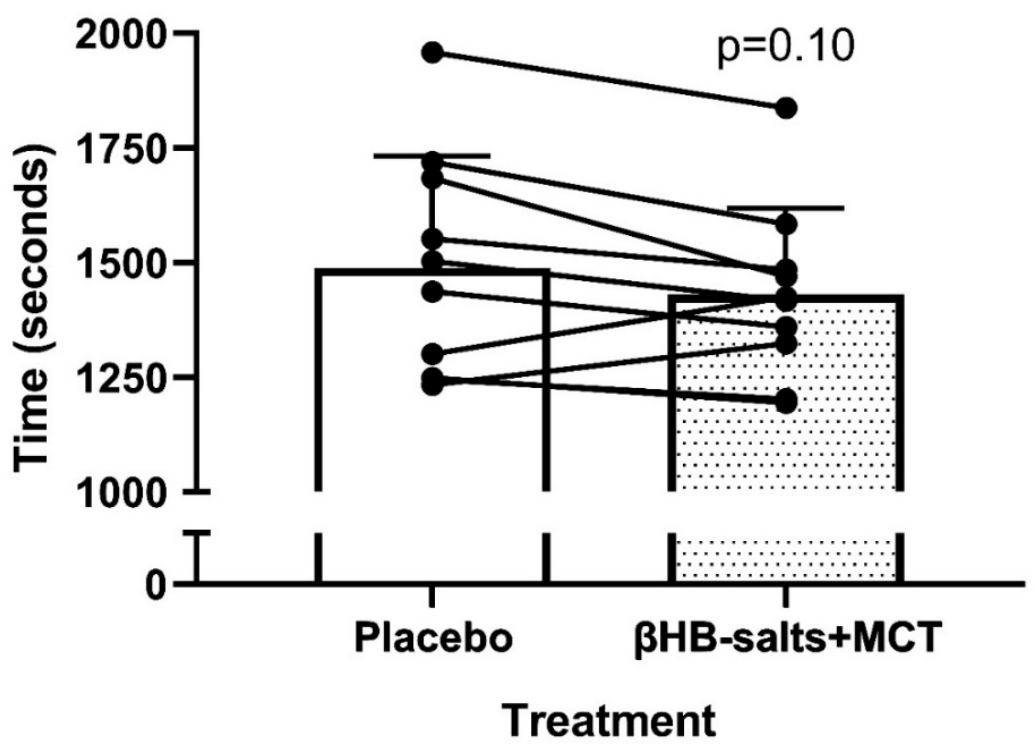

Figure 1

Mean and individual 5-km performance time following exogenous ketone supplement and placebo ingestion. 

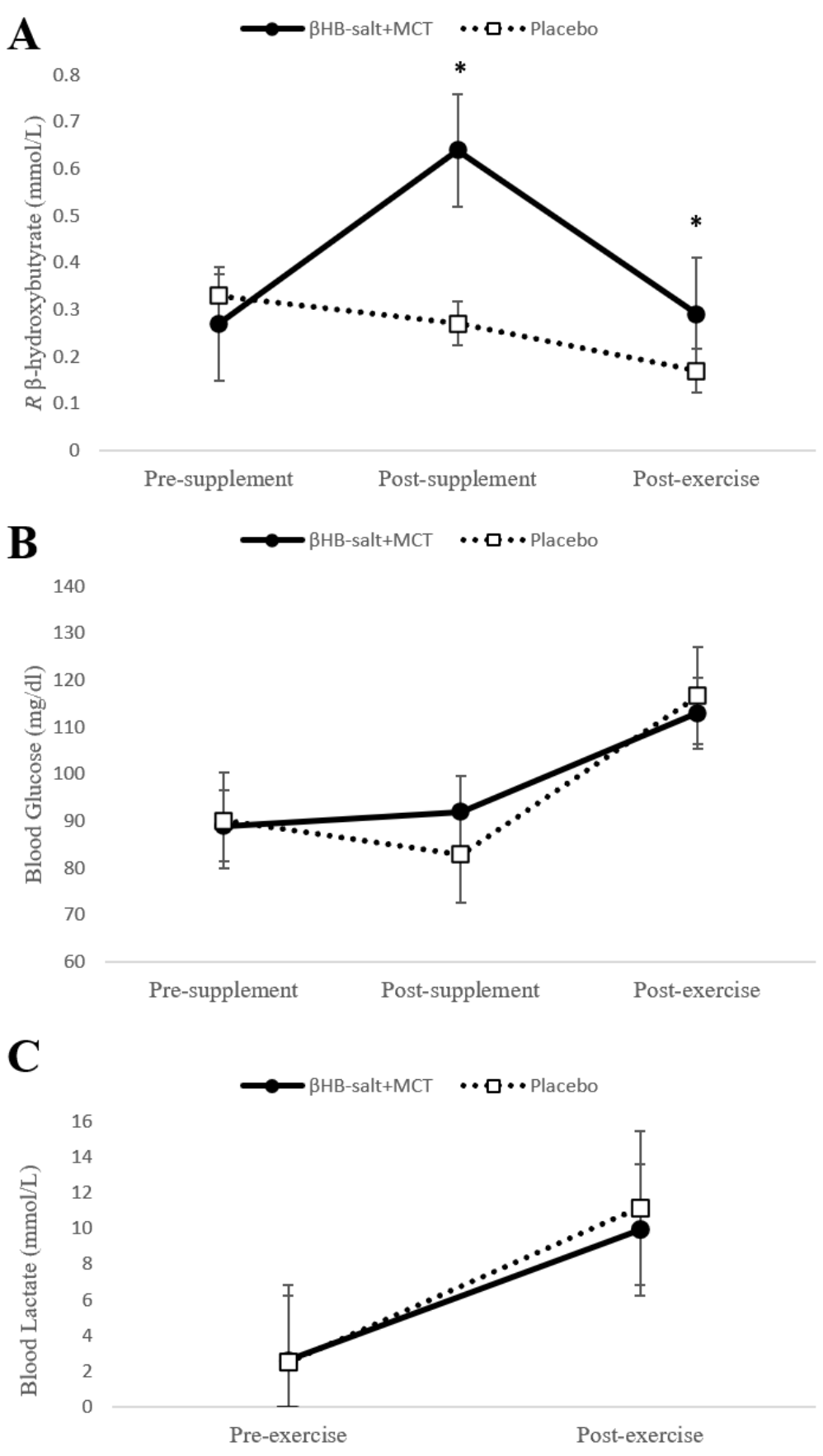

\section{Figure 2}

Impact of exogenous ketone supplementation on blood $R$ - $\beta$-hydroxybutyrate, glucose, and lactate. A) Blood $R$ - $\beta$-hydroxybutyrate was higher 60 min post-supplement and remained elevated throughout exercise in the oral ketone condition. B) Blood glucose rose during exercise but was not affected by oral ketone supplementation. C) Blood lactate rose during exercise, but was not affected by oral ketone supplementation. Values are Mean $\pm S D$.

$(N=10)$. ${ }^{*}$ Significant difference between Ketone and Placebo conditions $(p<0.05)$. 


\section{Physiological, Metabolic and Perceptual \\ Responses}

Blood $R-\beta H B$ increased from baseline under the $\beta \mathrm{HB}$-salt+MCT condition, and levels were elevated throughout the entire protocol compared to the PLA ( $p<0.05$ for all; Figure 2A). Blood lactate and glucose concentration rose from baseline to the end of exercise $(p<0.001)$, but there were no significant differences seen between conditions at any time point $(p>0.05$; Figure 2B and $\mathrm{C}$ ). Heart rate and RPE (chest, legs, and overall) increased significantly during exercise ( $p$ $<0.001)$, while affect deceased significantly during exercise $(p<0.001)$, however, there were no significant effects for treatment for any of the aforementioned variables $(p>0.05)$. In addition, no significant differences between the $\beta \mathrm{HB}-$ salt+MCT supplement and the PLA were observed for average HR $(173.1 \pm 15.7$ and $178.8 \pm$ $7.9 \mathrm{bpm}$, respectively; $p=0.322 ; \mathrm{ES}=0.46)$, average $\mathrm{VO}_{2}\left(3.0 \pm 0.5\right.$ and $3.0 \pm 0.5 \mathrm{~L} \cdot \mathrm{min}^{-1}$, respectively; $p$ $=0.665 ; \mathrm{ES}=0.08)$, average $\mathrm{VCO}_{2}(3.0 \pm 0.6$ and 2.9 $\pm 0.6 \mathrm{~L} \cdot \mathrm{min}^{-1}$, respectively; $\left.p=0.521 ; \mathrm{ES}=0.12\right)$, average RER (0.99 \pm 0.04 and $0.98 \pm 0.04$, respectively; $p=0.655 ; \mathrm{ES}=0.24)$, average $\mathrm{VE}(70.9$ \pm 14.8 and $69.4 \pm 15.0 \mathrm{~L} \cdot \mathrm{min}^{-1}$, respectively; $p=$ $0.607 ; \mathrm{ES}=0.10)$, average affect $(2.3 \pm 1.8$ and $1.8 \pm$ 1.8 , respectively; $p=0.143$; $\mathrm{ES}=0.28)$, average $\mathrm{RPE}-\mathrm{O}(5.5 \pm 1.3$ and $5.3 \pm 1.4$, respectively; $p=$ 0.593 ; ES $=0.15)$, average session RPE $(6.2 \pm 1.3$ and $7.3 \pm 1.6$, respectively; $p=0.093 ; 0.76)$, and average session affect $(2.6 \pm 1.6$ and $2.0 \pm 2.3$, respectively; $p=0.456 ; \mathrm{ES}=0.30$ ) (Table 2 ).

\section{Discussion}

The purpose of this study was to determine whether a commercially available exogenous ketone mixture of $\beta \mathrm{HB}$-salt+MCT would impact physiologic, metabolic, perceptional, and performance responses to intermediate distance running performance in recreational, college-age endurance athletes. We hypothesized that the $\beta \mathrm{HB}$-salt+MCT drink would alter blood metabolites and metabolism, but neither perception nor athletic performance. This is the first study to examine the effect of a commercially available ketone supplement on 5$\mathrm{km}$ running performance in recreational male distance runners. The major finding of this study was that ingesting $300 \mathrm{mg} \cdot \mathrm{kg}^{-1}$ body mass of an $\beta$ HB-salt+MCT drink $60 \mathrm{~min}$ before exercise resulted in a non-significant $4.06 \%$ improvement in $5-\mathrm{km}$ treadmill running time $(p=0.100)$. Furthermore, low variability of $5-\mathrm{km}$ time trials established in our laboratory and among endurance-trained distance runners (typical error of measurement of $31.75 \mathrm{~s}$ with a coefficient of variation of $2.18 \%$ ) suggests that altered endurance performance likely resulted from the ketone supplement ingestion (Prins et al., 2016). Additional analysis did show significant differences in circulating $R-\beta H B$, with no differences in other metabolic, physiologic, and perception variables. Together, results indicate a mixed response across participants after consuming $\quad 300 \quad \mathrm{mg} \cdot \mathrm{kg}^{-1} \quad \beta \mathrm{HB}$-salt+MCT supplement prior to exercise with $80 \%$ deemed responders and $20 \%$ non-responders.

Exogenous ketone supplements have emerged as a powerful and viable tool for regulating metabolism. Exogenous ketone formulations have been shown to safely raise circulating ketone levels and alter blood glucose (Kesl et al., 2016). This analysis looked at $\beta \mathrm{HB}-$ salt, MCT, $\beta \mathrm{HB}$-salt+MCT, ketone ester, and 1,3butanediol, demonstrating differential metabolic responses across the various exogenous ketone formulations tested. Ketone esters are widely considered the most potent exogenous ketone supplement and various agents are starting to enter the commercial market. 1,3-butanediol acetoacetate diester, a ketone ester, has recently been shown to reduce body mass and adiposity in obese mice, supporting the idea that ketones change energy metabolism (Davis et al., 2018). MCTs are naturally occurring in a variety of animal and vegetable fat, but can be consumed in a supplemental form. MCTs are ingested, shuttled to the hepatocyte inner mitochondrial membrane, and readily converted to $R-\beta \mathrm{HB}$ since they do not require carnitine to undergo $\beta$-oxidation (Bach et al., 1996; Sarda et al., 1987). $\beta$ HB-salts are typically in a racemic mixture of $50 \% R-\beta H B$ and $50 \% S$ $\beta \mathrm{HB}$ (Stubbs et al., 2017). To synthesize these molecules, $\beta \mathrm{HB}$ is ionically bound to monovalent or divalent cations $\left(\mathrm{Na}^{+}, \mathrm{K}^{+}, \mathrm{Mg}^{2+}, \mathrm{Ca}^{2+}\right)$ to create a salt. Thus, $\beta \mathrm{HB}$-salts accompany a mineral load depending on the choice and proportion of cation used to synthesize the salt. Upon ingestion, $\beta \mathrm{HB}-$ salts are absorbed through intestinal MCT transporters and enter systemic circulation where they can be readily metabolized in extra-hepatic 
tissue. $S-\beta \mathrm{HB}$ appears to undergo differential metabolic pathways than $R-\beta \mathrm{HB}$ (Desrochers et al., 1992; Stubbs et al., 2017, 2018) but does have some similar signaling capabilities (Newman and Verdin, 2017). $\beta$ HB-salt+MCT extends the circulating elevation of $R-\beta \mathrm{HB}$ further than when $\beta$ HB-salt-only is administered (Kesl et al., 2016). Unsurprisingly,

$\beta$ HB-salt+MCT significantly elevated circulating $R$ - $\beta \mathrm{HB}$ levels 2 fold $(0.3$ to $0.6 \mathrm{mM})$ in our findings. Similar elevations in circulating $R-\beta \mathrm{HB}$ have been seen in other trials with $\beta$ HB-salt supplementation (Evans et al., 2018; O'Malley et al., 2017; Rodger et al., 2017; Stubbs et al., 2017; Waldman et al., 2018). However, total $\beta \mathrm{HB}$ levels reported across these trails and ours, underestimate the total circulating $\beta \mathrm{HB}$ levels, as $S-\beta \mathrm{HB}$ is undetectable by current meter and bench top assay kits (Evans et al., 2018; O'Malley et al., 2017; Rodger et al., 2017; Waldman et al., 2018) and requires mass spectrum analysis (Stubbs et al., 2018). Stubbs et al. (2017) demonstrated that when a racemic $\beta \mathrm{HB}$-salt compound is orally administered, both $R-\beta \mathrm{HB}$ and $S-\beta H B$ become elevated, but $S-\beta H B$ is elevated to a higher degree due to its believed delayed metabolism. Thus, our results and others using $\beta \mathrm{HB}$-salts underestimate total $\beta \mathrm{HB}$ (Evans et al., 2018; O'Malley et al., 2017; Rodger et al., 2017; Waldman et al., 2018). While knowing total $\beta \mathrm{HB}$ is important for predicted systemic effect and cellular signaling, $S-\beta \mathrm{HB}$ is not fully metabolized through the ketolysis pathway and may not be consequential on the energetics of exercise performance in the present study (Stubbs et al., 2018). S- $\beta$ HB may play an important role in signaling that is being investigated for its protective effects (Newman and Verdin, 2017).

$R-\beta \mathrm{HB}$ elevations were observed in all studies involving $\beta$ HB-salts (Evans et al., 2018; O'Malley et al., 2017; Rodger et al., 2017; Stubbs et al., 2017; Waldman et al., 2018), including our present findings, but were substantially lower than those achieved via ketone esters (Cox et al., 2016; Evans and Egan, 2018; Leckey et al., 2017; Stubbs et al., 2017). Additionally, serum glucose levels did not substantially change in the current study compared to the placebo, a finding that has been previously reported with ingestion of $\beta \mathrm{HB}-$ salts (Rodger et al., 2017; Waldman et al., 2018). However, this is not consistently reported (O'Malley et al., 2017; Stubbs et al., 2017). Lactate is a substrate biomarker for glycolytic metabolism and is commonly elevated post-exercise. Initial report on ketone ester ingestion demonstrated lower lactate production during exercise (Cox et al., 2016), which was confirmed by two subsequent analyses of various ketone ester formulations (Evans and Egan, 2018; Leckey et al., 2017). However, this is dissimilar to the augmented (Volek et al., 2016) and neutral effects (Zajac et al., 2014) reported on exercise lactate production with VLCKD. Exogenous ketoneinduced attenuated lactate production was initially proposed as a glucose sparring mechanism which allowed for glucose to be present for anaerobic efforts at the end of a race (Cox et al., 2016). However, others have speculated on the potential for ketones to attenuate glucose utilization and subsequent lactate production, potentially inhibiting performance (Evans and Egan, 2018; Leckey et al., 2017). Some argue that this may be a consequence of changes seen in pyruvate dehydrogenase activity as has been seen in low-carbohydrate dietary regimens (Stellingwerff et al., 2006). However, it is currently unknown whether changes in pyruvate dehydrogenase activity occur consistently with acute ketone administration and whether this will influence performance. Additionally, an attenuation of lactate production has not been reported in any trial using $\beta$ HB-salts (Evans et al., 2018; O'Malley et al., 2017; Rodger et al., 2017; Waldman et al., 2018), which agrees with our findings and those by Zajac et al. (2014) using a VLCKD. Taken together, our metabolic data indicate that consumption of $\beta \mathrm{HB}$-salt+MCT results in significantly elevated circulation of $\beta \mathrm{HB}$, a finding that is reported across all exogenous ketone supplements (Stubbs et al., 2017; Leckey et al., 2017). However, we did not find changes in blood glucose levels, which is consistent with some reports using $\beta \mathrm{HB}$-salt (Rodger et al., 2017; Waldman et al., 2018), and inconsistent with others (O'Malley et al., 2017; Stubbs et al., 2017). Together, these results indicate mixed results on the blood glucose lowering effects of $\beta \mathrm{HB}$-salt administration. Additionally, the unchanged lactate levels reported in the present study indicate that higher levels of circulating ketones may be required to attenuate lactate production, an effect that has been seen with more potent forms of exogenous 
ketone administration (Cox et al., 2016; Evans and Egan, 2018; Leckey et al., 2017), but not found in $\beta$ HB-salt administration (Evans et al., 2018; O'Malley et al., 2017; Rodger et al., 2017; Waldman et al., 2018). Interestingly, the augmented (Volek et al., 2016) and neutral effects (Zajac et al., 2014) of a VLCKD on exercise lactate production highlight potential metabolic overlap and discrepancies between a VLCKD and various exogenous ketone formulations.

Substrate utilization is commonly extrapolated from the amount of $\mathrm{O}_{2}$ consumed and $\mathrm{CO}_{2}$ expelled (Jeukendrup and Wallis, 2005). Previous analyses of $\beta \mathrm{HB}$-salt under such calculations were extrapolated to indicate that $\beta$ HB-salts may increase fat-oxidation (O'Malley et al., 2017). However, such assumptions during ketone supplementation without adjustment calculations may not be valid (Evans and Egan, 2018). Equating substrate utilization from RER measurements cannot be reliably done without adjusting calculations for ketone supplementation as RER of $\beta \mathrm{HB}$ and AcAc are 0.89 and 1.00, respectively, directly confounding calculations of glucose and fat oxidation (Frayn, 1983). Thus, substrate level utilization cannot be determined from the present study or others testing $\beta \mathrm{HB}$-salts (O'Malley et al., 2017). Additionally, while RER was not significantly different within the current study, RER has had inconsistent results in previous analysis involving $\beta \mathrm{HB}$-salts with some indicating lower RER (O'Malley et al., 2017), and some indicating higher (Evans et al., 2018; Rodger et al., 2017).

Finally, we did not find a performanceenhancing effect across the mean of all 10 participants, although a non-significant decrease in time to completion of $4.06 \%$ ( 60 seconds) was observed $(p=0.100)$. To date, only three studies have analyzed $\beta \mathrm{HB}$-salts as an ergogenic or ergolytic supplement in performance outcomes:

1. a 15-min staged exercise regimen prior to a $10 \mathrm{~km}$ cycling time trial (O'Malley et al., 2017).

2. a 90 min steady-state bout of cycling at $80 \%$ of the ventilatory threshold, followed by a $4-\mathrm{km}$ time trial (Rodger et al., 2017)

3. a $5 \mathrm{~min}$ warm up prior to four $15 \mathrm{~s}$ maximum effort intervals (Waldman et al., 2018).

Two of the three trials showed no difference when aerobic activity was used prior to an analyzed intense anaerobic environment thereafter, both using various cycling exercise regimens (Rodger et al., 2017; Waldman et al., 2018). However, one of the trials showed a $6.9 \%$ slower time and $7 \%$ lower power output during time trial completion using graded cycling exercise $(30 \%, 60 \%, 90 \%$ of ventilatory threshold) followed by a measured $10-\mathrm{km}$ time trial (O'Malley et al., 2017), with two subjects performing worse. However, our data indicate a mixed response across participants consuming the $\beta \mathrm{HB}$-salt+MCT combination.

One potential explanation for the alternative response reported is the type of exercise regimen utilized in the present analysis, i.e., running. Alternatively, all previous analysis into $\beta \mathrm{HB}$-salts with neutral or negative effects utilized cycling as exercise of choice (O'Malley et al., 2017; Rodger et al., 2017; Waldman et al., 2018), indicating potential supplemental sport ergogenic specificity. A more likely explanation for the result discrepancy in the present trial is the lack of preliminary exhaustive exercise prior to the $5-\mathrm{km}$ time trial. The likely rational for providing exhaustive exercise prior to a time trial performance is to replicate the race environment where the final stretch of the race requires all out (timed) effort and thus can have influence on the final race performance and finish. Experimental designs may also be influenced by the original work done in cyclists using the ketone ester which involved a preliminary steady state exercise regimen for $60 \mathrm{~min}$ followed by a 30-min time trial (Cox et al., 2016). Additionally, the original analysis of ketone ester supplementation indicated that $>1.0 \mathrm{mM}$ elevations in ketone may attenuate glucose utilization or be glycogen sparring (Cox et al., 2016). However, glucose sparing effect of ketones only appears to happen in the context of ketone ester as they all have shown attenuated lactate production (Cox et al., 2016; Evans and Egan, 2018; Leckey et al., 2017). Lastly, we cannot exclude the possibility that this unique formulation contributed to discrepant performance outcomes when compared with pure racemic $\beta \mathrm{HB}$-salts as this $\beta \mathrm{HB}-$ salt+MCT formulation contains a higher percentage of $R$ $\beta \mathrm{HB}$ precursors (Kesl et al., 2016), has shown to produce a more prolonged elevations in circulating serum $R-\beta \mathrm{HB}$ in rodents, and all 
historical $R-\beta \mathrm{HB}$ analyses in exercised human cohorts demonstrating similar $R-\beta H B$ elevations are limited to timepoint measurements and not total $R-\beta \mathrm{HB}$ exposure over study duration (O'Malley et al., 2017; Rodger et al., 2017; Waldman et al., 2018).

Limitations of the present study include the method of plasma metabolite analysis, inability to differentiate this supplement used against other fueling strategies, and the inability to directly measure tissue substrate utilization to determine tissue specific effects. Discrepancies have been observed across handheld meters and bench top assays, neither of which assessed total $\beta \mathrm{HB}$ levels in the blood. Mass spectrum analysis is the only method currently available to determine $S-\beta \mathrm{HB}$ and total $\beta \mathrm{HB}$. The present study was conducted to determine the ergolytic or ergogenic influence of a specific formula of $\beta \mathrm{HB}-$ salts+MCT. Consequently, we are unable to determine whether supplementing with $\beta \mathrm{HB}-$ salts+MCT is superior to other known athletic fuel strategies. Lastly, RER measurements are traditionally used to determine fat to carbohydrate oxidation. However, ketone supplementation confounds these measurements and would require a combination of ketone excretion and blood ketone measurements to estimate influence of ketones on substrate level oxidation.

\section{Conclusions}

Traditionally, ketosis was only achieved through dietary manipulation. Recent interest has arisen to develop ketone bodies that can be exogenously administered without dietary carbohydrate restriction. With the emergence of commercially available exogenous ketone formulations, research has focused on exploring their use in athletic performance. To our knowledge this is the first study to report the effects of oral ketone supplement ingestion on 5- $\mathrm{km}$ treadmill running performance. This investigation demonstrated that recreational endurance athletes consuming a $\beta \mathrm{HB}$-salt $+\mathrm{MCT}$ formula showed a non-significant $4.06 \%$ decrease in time to complete volitional 5$\mathrm{km}$ run performance $(p=0.100)$. $\beta \mathrm{HB}$-salt+MCT consumption did significantly increase blood $R$ $\beta \mathrm{HB}$ levels, with no differences in other metabolic, physiologic, and perception variables. Results indicate that runners may experience mixed responses to consuming a $300 \mathrm{mg} \cdot \mathrm{kg}^{-1} \beta \mathrm{HB}-$ salt+MCT supplement prior to exercise. Further testing will be needed to determine if $\beta \mathrm{HB}$ salts+MCT provides a unique metabolic advantage, and if this altered response is specific to the exercise task.

\section{Acknowledgements}

The study was funded by the Grove City College Exercise Science Department. The authors wish to thank the participants for their vital contribution to this study.

\section{Conflicts of interest}

DD is an inventor on a patent entitled "Composition and Methods of Elevating and Sustaining Ketosis" USPTO\# 20170266148. This invention was made with government support under Grant \# N0001413-1-0062 awarded by the Department of Defense, Office of Naval Research. AK and DD are inventors on provisional patents "Compositions and Methods for Weight Loss Maintenance" and "Prevention of Muscle Wasting with Ketone Supplementation" using technologies dissimilar to the ketones formulation used in this manuscript. At the time of this publication, provision patents were still under review. However, should provisional patents become accepted and royalties ever accrue, AK and DD will receive a share under the terms prescribed by the University of South Florida. DD is an owner of Ketone Technologies LLC and has served as a consultant and conference speaker.

\section{References}

Astorino TA, Matera AJ, Basinger J, Evans M, Schurman T, Marquez R. Effects of red bull energy drink on repeated sprint performance in women athletes. Amino Acids, 2012; 42: 1803-1808

Bach AC, Ingenbleek Y, Frey A. The usefulness of dietary medium-chain triglycerides in body weight control: fact or fancy? J Lipid Res, 1996; 37: 708-726 
Cahill GF, Jr. Fuel metabolism in starvation. Annu Rev Nutr, 2006; 26: 1-22

Cox PJ, Kirk T, Ashmore T, Willerton K, Evans R, Smith A, Murray AJ, Stubbs B, West J, McLure SW, King MT, Dodd MS, Holloway C, Neubauer S, Drawer S, Veech RL, Griffin JL, Clarke K. Nutritional Ketosis Alters Fuel Preference and Thereby Endurance Performance in Athletes. Cell Metab, 2016; 24: 256-268

Dansinger ML, Gleason JA, Griffith JL, Selker HP, Schaefer EJ. Comparison of the Atkins, Ornish, Weight Watchers, and Zone diets for weight loss and heart disease risk reduction: a randomized trial. JAMA, 2005; 293: 43-53

Davis RAH, Deemer SE, Bergeron JM, Little JT, Warren JL, Fisher G, Smith DL, Jr., Fontaine KR, Dickinson SL, Allison DB, Plaisance EP. Dietary R, S-1,3-butanediol diacetoacetate reduces body weight and adiposity in obese mice fed a high-fat diet. FASEB, 2018; 33: 2409-2421

Desrochers S, David F, Garneau M, Jette M, Brunengraber H. Metabolism of R- and S-1,3-butanediol in perfused livers from meal-fed and starved rats. Biochem J, 1992; 285 (Pt 2): 647-653

Evans M, Egan B. Intermittent Running and Cognitive Performance after Ketone Ester Ingestion. Med Sci Sports Exerc, 2018; 50: 2330-2338

Evans M, Patchett E, Nally R, Kearns R, Larney M, Egan B. Effect of acute ingestion of beta-hydroxybutyrate salts on the response to graded exercise in trained cyclists. Eur J Sport Sci, 2018; 18: 376-386

Forbes SC, Candow DG, Little JP, Magnus C, Chilibeck PD. Effect of Red Bull energy drink on repeated Wingate cycle performance and bench-press muscle endurance. Int J Sport Nutr Exerc Metab, 2007; 17: 433-444

Frayn KN. Calculation of substrate oxidation rates in vivo from gaseous exchange. J Appl Physiol Respir Environ Exerc Physiol, 1983; 55: 628-634

Hardy CJR, W.J. Not what, but how one feels: The measurement of affect during exercise. J Sport Exerc Psychol, 1989; 11: 304-317

Jeukendrup AE, Wallis GA. Measurement of substrate oxidation during exercise by means of gas exchange measurements. Int J Sports Med, 2005; 26 Suppl 1: S28-37

Kesl SL, Poff AM, Ward NP, Fiorelli TN, Ari C, Van Putten AJ, Sherwood JW, Arnold P, D'Agostino DP. Effects of exogenous ketone supplementation on blood ketone, glucose, triglyceride, and lipoprotein levels in Sprague-Dawley rats. Nutr Metab, 2016; 13: 9

Koutnik AP, D'Agostino DP, Egan B. Anticatabolic Effects of Ketone Bodies in Skeletal Muscle. Trends Endocrinol Metab, 2019; doi: 10.1016/j.tem.2019.01.006

Leckey JJ, Ross ML, Quod M, Hawley JA, Burke LM. Ketone Diester Ingestion Impairs Time-Trial Performance in Professional Cyclists. Front Physiol, 2017; 8: 806

Mascioli EA, Randall S, Porter KA, Kater G, Lopes S, Babayan VK, Blackburn GL, Bistrian BR. Thermogenesis from intravenous medium-chain triglycerides. J Parenter Enteral Nutr, 1991; 15: 27-31

Michalczyk MM, Chycki J, Zajac A, Maszczyk A, Zydek G, \& Langfort J. Anaerobic Performance after a Low-Carbohydrate Diet (LCD) Followed by 7 Days of Carbohydrate Loading in Male Basketball Players. Nutrients, 2019; doi: 10.3390/nu11040778

Newman JC, Covarrubias AJ, Zhao M, Yu X, Gut P, Ng CP, Huang Y, Haldar S, Verdin E. Ketogenic Diet Reduces Midlife Mortality and Improves Memory in Aging Mice. Cell Metab, 2017; 26: 547-557 e548

Newman JC, Verdin E. Beta-Hydroxybutyrate: A Signaling Metabolite. Annu Rev Nutr, 2017; 37: 51-76

O'Malley T, Myette-Cote E, Durrer C, Little JP. Nutritional ketone salts increase fat oxidation but impair high-intensity exercise performance in healthy adult males. Appl Physiol Nutr Metab, 2017; 42: 10311035

Paoli A, Grimaldi K, D'Agostino D, Cenci L, Moro T, Bianco A, Palma A. Ketogenic diet does not affect strength performance in elite artistic gymnasts. J Int Soc Sports Nutr, 2012; 9: 34

Paoli A, Rubini A, Volek JS, Grimaldi KA. Beyond weight loss: a review of the therapeutic uses of very-lowcarbohydrate (ketogenic) diets. Eur J Clin Nutr, 2013; 67: 789-796

Phinney SD, Bistrian BR, Evans WJ, Gervino E, Blackburn GL. The human metabolic response to chronic ketosis without caloric restriction: preservation of submaximal exercise capability with reduced carbohydrate oxidation. Metabolism, 1983a; 32: 769-776

Phinney SD, Bistrian BR, Wolfe RR, Blackburn GL. The human metabolic response to chronic ketosis without 
caloric restriction: physical and biochemical adaptation. Metabolism, 1983b; 32: 757-768

Phinney SD, Horton ES, Sims EA, Hanson JS, Danforth E, Jr., LaGrange BM. Capacity for moderate exercise in obese subjects after adaptation to a hypocaloric, ketogenic diet. J Clin Invest, 1980; 66: 1152-1161

Poff A, Koutnik AP, Egan KM, Sahebjam S, D'Agostino D, Kumar NB. Targeting the Warburg effect for cancer treatment: Ketogenic diets for management of glioma. Semin Cancer Biol, 2017; doi: 10.1016/j.semcancer.2017.12.011

Prins PJ, Goss FL, Nagle EF, Beals K, Robertson RJ, Lovalekar MT, Welton GL. Energy Drinks Improve FiveKilometer Running Performance in Recreational Endurance Runners. J Strength Cond Res, 2016; 30: 2979-2990

Roberts MN, Wallace MA, Tomilov AA, Zhou Z, Marcotte GR, Tran D, Perez G, Gutierrez-Casado E, Koike S, Knotts TA, Imai DM, Griffey SM, Kim K, Hagopian K, McMackin MZ, Haj FG, Baar K, Cortopassi GA, Ramsey JJ, Lopez-Dominguez JA. A Ketogenic Diet Extends Longevity and Healthspan in Adult Mice. Cell Metab, 2017; 26: 539-546 e535

Robertson RJ, Goss FL, Dube J, Rutkowski J, Dupain M, Brennan C, Andreacci J. Validation of the adult OMNI scale of perceived exertion for cycle ergometer exercise. Med Sci Sports Exerc, 2004; 36: 102-108

Rodger S, Plews D, Laursen P, Driller M. Oral B-Hydroxybutyrate salt fails to improve 4-minute cycling performance following submaximal exercise. J Sci Cycling, 2017; 6: 26-31

Sarda P, Lepage G, Roy CC, Chessex P. Storage of medium-chain triglycerides in adipose tissue of orally fed infants. Am J Clin Nutr, 1987; 45: 399-405

Seaton TB, Welle SL, Warenko MK, Campbell RG. Thermic effect of medium-chain and long-chain triglycerides in man. Am J Clin Nutr, 1986; 44: 630-634

Stafstrom CE, Rho JM. The ketogenic diet as a treatment paradigm for diverse neurological disorders. Front Pharmacol, 2012; 3: 59

Stellingwerff T, Spriet LL, Watt MJ, Kimber NE, Hargreaves M, Hawley JA, Burke LM. Decreased PDH activation and glycogenolysis during exercise following fat adaptation with carbohydrate restoration. Am J Physiol Endocrinol Metab, 2006; 290: E380-388

Stubbs BJ, Cox PJ, Evans RD, Santer P, Miller JJ, Faull OK, Magor-Elliott S, Hiyama S, Stirling M, Clarke K. On the Metabolism of Exogenous Ketones in Humans. Front Physiol, 2017; 8: 848

Stubbs BJ, Koutnik AP, Poff AM, Ford KM, D'Agostino DP. Commentary: Ketone Diester Ingestion Impairs Time-Trial Performance in Professional Cyclists. Front Physiol, 2018; 9: 279

Volek JS, Freidenreich DJ, Saenz C, Kunces LJ, Creighton BC, Bartley JM, Davitt PM, Munoz CX, Anderson JM, Maresh CM, Lee EC, Schuenke MD, Aerni G, Kraemer WJ, Phinney SD. Metabolic characteristics of keto-adapted ultra-endurance runners. Metabolism, 2016; 65: 100-110

Waldman HS, Basham SA, Price FG, Smith JW, Chander H, Knight AC, Krings BM, McAllister MJ. Exogenous ketone salts do not improve cognitive responses after a high-intensity exercise protocol in healthy college-aged males. Appl Physiol Nutr Metab, 2018; 43: 711-717

Wilson JM, Lowery RP, Roberts MD, Sharp MH, Joy JM, Shields KA, Partl J, Volek JS, D'Agostino D. The Effects of Ketogenic Dieting on Body Composition, Strength, Power, and Hormonal Profiles in Resistance Training Males. J Strength Cond Res, 2017; doi: 10.1519/JSC.0000000000001935

Zajac A, Poprzecki S, Maszczyk A, Czuba M, Michalczyk M, Zydek G. The effects of a ketogenic diet on exercise metabolism and physical performance in off-road cyclists. Nutrients, 2014; doi: $10.3390 /$ nu6072493

\section{Corresponding author:}

\section{Philip Prins, Ph.D.}

Department of Exercise Science

100 Campus Drive, Grove City College, Grove City, PA 16127

Telephone: (724) 458-3863

E-mail: pjprins@gcc.edu 